\title{
Novel Dysregulated MicroRNAs in Primary Laryngeal Squamous Cell Cancer
}

\author{
Kang Mei Chen, Josena K Stephen, Shaleta Havard, Veena Shah, Glendon Gardner
}

Vanessa G Schweitzer, Maria J Worsham

\begin{abstract}
Introduction: MicroR NAs (miRNAs) are endogenous, small, noncoding RNAs of 17 to 25 nucleotides that regulate approximately $30 \%$ of human genes. They are differentially expressed in various types of cancers compared with noncancerous tissues, suggesting that they may have crucial roles in tumorigenesis. The objective of this study was to identify laryngeal squamous cell cancer (LSCC)-specific miR NAs.
\end{abstract}

Materials and methods: A retrospective cohort of 10 LSCC and five normal laryngeal squamous epithelium samples were examined using a global miRNA profiling approach (HTG, Tucson, AZ, USA, 800 human miRNAs plus 10 endogenous control miRNAs). The expression status of selected dysregulated miRNAs that were significantly different from normal were verified by real-time quantitative PCR ( $P P C R$ ).

Results: Twenty-three of the 800 human miRNAs had significantly different expression levels $(p<0.05)$ between LSCC and normal tissues. Fifteen of the 23 have not been previously reported in HNS CC and include: miR -663b, miR-663, miR -193b, miR-1291, miR-720, miR-191, miR-1224-3p, miR-214, miR1285, miR-1207-5p, miR-483-5p, miR-1225-3p, miR-1228, miR 1280 and miR -638. Consistently upregulated miR -31 and miR 193b and differentially expressed miR-663b in LSCC were verified by $\mathrm{qPCR}$.

Conclusion: The 15 novel miR NAs identified in this exploratory study, pending further confirmation and validation, may have clinical utility as LSCC-specific markers.

Keywords: MicroRNAs, Laryngeal squamous cell cancer, Global microR NA profiling, qPCR validation.

How to cite this article: Chen KM, Stephen J K, Havard S Shah V, Gardner G, Schweitzer VG, Worsham MJ. Novel Dysregulated MicroR NAs in P rimary Laryngeal Squamous Cell Cancer. Int J Head Neck Surg 2012;3(2):76-81.

\section{Source of support: $\mathrm{Nil}$}

\section{Conflict of interest: None}

\section{INTRODUCTION}

MicroRNAs (miRNAs) are endogenous, small, noncoding RNA s of 17 to 25 nucleotides that are thought to regulate approximately $30 \%$ of human genes. ${ }^{1-3}$ They are involved in regulating target gene expression through imperfect base pairing with the 3'-untranslated region (3'-UTR) of target mRNAs of protein-coding genes, leading to the cleavage of homologous mRNA or translational inhibition. They are differentially expressed in various types of cancers compared with noncancerous tissues, suggesting that they may have crucial roles in tumorigenesis. ${ }^{4-8}$
Quite a few miR N A s have been linked to head and neck squamous cell carcinoma (HNSCC) based on their differential expression in tumors. miR-21, a commonly dysregulated miRNA in cancer, is frequently upregulated in HNSCC. ${ }^{9-14}$ Progression of oral cancers has been significantly associated with miR-345, -21 and $-181 b^{12}$ Significantly low levels of miR-125a and miR-200a have been detected in the saliva of oral cancer patients ${ }^{15}$ while high levels of miR-184 have been detected in the plasma of tongue SCC. ${ }^{16} \mathrm{~A}$ Itered expression of specific miR NAs are beginning to provide much needed insights into tumorigenesis mechanisms of abnormal cell-cycle regulation, evasion of apoptosis, reduced response to antigrowth signals and epithelial-mesenchymal transition (EM T). ${ }^{17}$ These reports strongly support the potential utility of miRNAs as diagnostic biomarkers in HNSCC. The objective of this pilot study was to identify laryngeal squamous cell carcinoma (LSCC)-specific miRNA s using a global discovery approach.

\section{MATERIALS AND METHODS}

\section{Cohort}

The retrospective pil ot cohort comprised 10 primary LSCC cases (cancer cohort) and five normal laryngeal squamous epithelium tissues (control cohort). This study was approved by the Henry Ford Health System Institutional Review B oard Committee.

\section{RNA Extraction}

miRNA from tumor and normal tissue, present in separate formalin-fixed, paraffin-embedded (FFPE) tissue blocks was extracted using the High pure miR NA Isolation K it (Roche). Final dilution of total RNA used for reverse transcription was $20 \mathrm{ng} / \mathrm{ul}$.

\section{Quantitative Nuclease Protection miRNA Microarray Assay}

Global miRNA profiling using quantitative nuclear protection (qNPA) miRNA microarray assays was provided by High Throughput Genomics Inc. (HTG, Tucson, AZ, USA). This assay interrogates a panel of 800 human miRNAs plus 10 endogenous control miRNAs simultaneously. ${ }^{18}$ We provided two curls of $5 \mu \mathrm{m}$ tissue sections in tubes for each of the 15 cases. 


\section{HTG Data Analysis}

Each microarray has two elements (replicates) for each transcript (A 1.1 and A 1.2, for example); therefore the total number of data points for a single transcript is 2 . Data is normalized to the total signal for each microarray. A particular transcript is considered expressed, if the signal is more than three standard deviations above the background signal as determined by the ANT gene.

Significantly differently expressing miRNAs between normal and tumor samples were obtained using the Student's t-test. Significantly $(p<0.05)$ upregulated and downregulated miRNAs detected in the LSCC group (as compared to the normal group) by the qN PA assay arelisted in Table 1.

Table 1: Significantly upregulated and downregulated miRNAs

\begin{tabular}{|c|c|c|c|}
\hline miR NA & Ratio & p-value & $\begin{array}{l}\text { Chromosome } \\
\text { location }\end{array}$ \\
\hline \multicolumn{4}{|l|}{ Upregulated } \\
\hline hsa-miR-320a & 4.81 & 0.000 & $8 p 21.3$ \\
\hline hsa-miR-320b & 4.32 & 0.000 & 1 \\
\hline hsa-miR-663 & 3.38 & 0.000 & $20 p 11.1$ \\
\hline hsa-miR-193b ${ }^{\dagger}$ & 10.83 & 0.001 & $16 \mathrm{p} 13.12$ \\
\hline hsa-miR-92a & 5.72 & 0.001 & $13 q 31.3 / X q 26.2$ \\
\hline hsa-miR-1291 & 3.98 & 0.001 & 12 \\
\hline hsa-miR-16 & 3.97 & 0.003 & $13 q 14.3 / 3 q 26.1$ \\
\hline hsa-miR-720 & 2.07 & 0.004 & 3 \\
\hline hsa-miR-27a & 5.11 & 0.005 & $19 \mathrm{p} 13.13$ \\
\hline hsa-let-7f & 2.89 & 0.006 & $9 q 22.32 / X p 11.22$ \\
\hline hsa-miR-191 & 3.30 & 0.009 & $3 p 21.31$ \\
\hline hsa-miR-1224-3p & 3.19 & 0.009 & $3 q 27.1$ \\
\hline hsa-miR- $31^{\dagger}$ & 6.16 & 0.010 & $9 p 21.3$ \\
\hline hsa-miR-214 & 3.15 & 0.010 & $1 q 24.3$ \\
\hline hsa-miR-1285 & 2.23 & 0.010 & $7 / 2$ \\
\hline \multicolumn{4}{|l|}{ Downregulated } \\
\hline hsa-miR-1207-5p & 0.61 & 0.000 & 8 \\
\hline hsa-miR-483-5p & 0.62 & 0.000 & $11 p 15.5$ \\
\hline hsa-miR-1280 & 0.76 & 0.000 & 3 \\
\hline hsa-miR-1228 & 0.76 & 0.001 & 12 \\
\hline hsa-miR-1225-3p & 0.76 & 0.002 & $16 p 13.3$ \\
\hline hsa-miR-296-5p & 0.75 & 0.008 & $20 q 13.32$ \\
\hline hsa-miR-638 & 0.83 & 0.035 & $19 p 13.2$ \\
\hline
\end{tabular}

${ }^{\dagger}$ Verified by qRT-PCR

Bolded: Previously unreported miR NAs

Five miRNAs with the lowest $p$-values (three upregulated: miR-663b, miR-193b, and miR-31; two downregulated: miR-923 and miR-1826) were selected for further verification by quantitative real-time PCR.

\section{TaqMan MicroRNA Reverse Transcription}

Reverse transcription was performed using TaqM an M icroR N A Reverse Transcription K it (A BI) which was used in conjunction with the TaqMan MicroRNA Individual A ssays ( $\mathrm{B} \mathrm{B}$ ) containing the individual reverse transcription primers. The manufacturer's prescribed protocol was followed for both kits. Final volume for 1 reaction is $7.5 \mathrm{ul}$ (3.5 ul RT master mix, 2.5 ul total RNA, and 1.5 ul RT primer).

\section{Real-Time Quantitative PCR (qPCR)}

Real-time quantitative PCR (qPCR) approach was employed to verify expression status of selected miRNAs that were significantly different from normal controls as proof-ofprinciple. This was performed using TaqM an Universal Master M ix II (no UNG). M anufacturer's protocol was followed for microRNA individual assays. Each samplewas run in duplicate.

\section{qPCR Data Analysis}

miRNA expression in the normal and tumor groups was measured using ${ }^{\Delta \Delta} \mathrm{C}$ t relative quantization. miRNA s with a value $<0$ were considered as downregulated and $>0$ were upregulated (Fig. 1).

\section{RESULTS}

All five normal samples and nine of $10 \mathrm{LSCC}$ cases were part of the analyses (one tumor sample indicated outlier values and was excluded). Twenty-three of the 800 human miRN A s were differentially expressed $(p<0.05)$ between LSCC and normal tissues (Table 1), of which 16 were upregulated and seven were downregulated.

Fifteen of the 23 dysregulated miRNA s have not been previously reported in HNSCC and include: miR-663b,

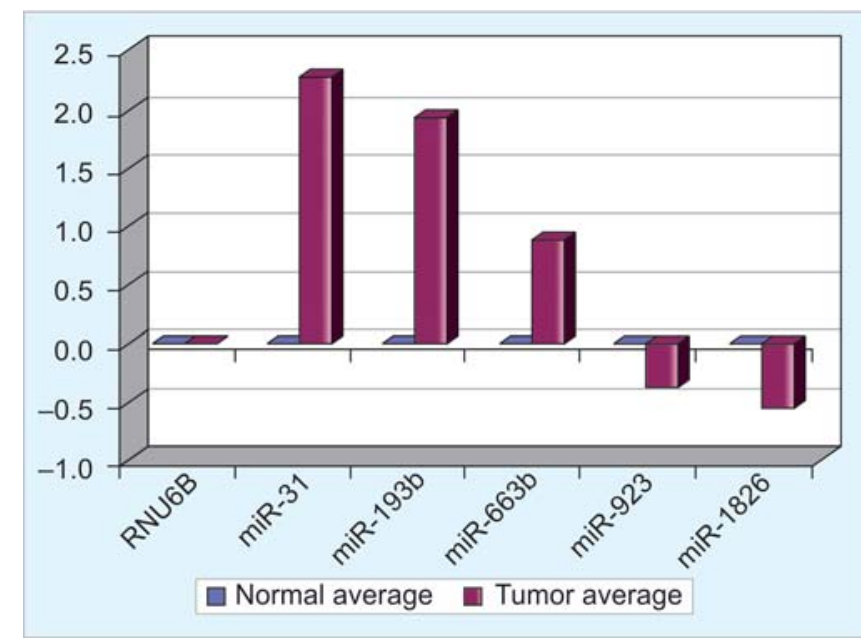

Fig. 1: qPCR data-miRNA expression levels in normal and tumor groups

RNU6B, the internal control miRNA, is not expressed in normal or tumor samples. miR-31, miR-193b and miR-663b are upregulated, whereas miR-923 and miR-1826 were downregulated in the tumor samples. miR-923 and miR-1826 are now listed as fragments in recent updates of the miRNA database and, therefore, not considered as miRNAs 
miR-663, miR-193b, miR-1291, miR-720, miR-191, miR1224-3p (3' arm of miR-1224 hairpin), miR-214, miR-1285, miR-1207-5p (5' arm of miR-1207 hairpin), miR-483-5p, miR-1225-3p, miR-1228, miR-1280 and miR-638 (highlighted in bold in Table 1).

Selected miRNAs, miR-923, miR-1826, miR-663b, miR-193b and miR-31, with p-values 0.000, 0.000, 0.000, 0.001 and 0.01 respectively, were verified by $\mathrm{QPCR}$ and were concordant with microarray data. miR-31 and miR193b were consistently upregulated (9/9), and miR-663b was differentially regulated (7/9 upregulated and $2 / 9$ downregulated) in LSCC (Table 2). miR-923 and miR-1826 (not shown in Table 1) showed downregulation in $6 / 9$ and upregulation in 3/9 samples; however, these are now listed as fragments in recent updates of the miRN A database and no longer regarded as legitimate miR NAs.

\section{DISCUSSION}

miR NAs, though lurking behind the scenes, are now being viewed less as molecular noise and more as exotic players with increasing prominence in theories about cancer. ${ }^{19} \mathrm{By}$ binding to $\mathrm{mRNA}$, miR NA can silence or modulate mRNA function. Expression profiling of miRNAs has shown that some miR N A s are upregulated or down-regulated in cancer, suggesting that it is important to understand the specific roles miRNA s may have in cancer.

There are two types of cancer-related miRNAs: Oncogenic (i.e. miR-155, miR-21) ${ }^{8}$ or tumor suppressor miRNAs (i.e. miR-15a and let-7 family). ${ }^{20,21}$ In HNSCC, miR N A profiling has been performed in oral, oropharyngeal, tongue, laryngeal and thyroid cancers. A study by $\mathrm{Hui}$ et $\mathrm{al}^{22}$ found no distinct differences in the global miRNA profiles between squamous cell cancers arising from the larynx, oropharynx or hypopharynx with the exception of miR-133b which was more highly expressed in laryngeal vs the other two subsites. They found about a third of the miRNAs examined in their HNSCC samples to be dysregulated.
Because several of the differentially expressed miR NAs in their study were in frequently amplified or deleted regions, genomic amplifications or deletions were offered as one possible mechanism for abnormal miRNA expression in HNSCC.

We found 23 significantly differentially expressed miRNA s for many of which, information is limited to chromosomal locations (Table 1). Of the 23 significantly differentially expressed miRNAs, eight have been reported previously in HNSCC. These include miR-320a, miR-320b, miR-92a, miR-16, miR-27a, let-7f, miR-31 and miR-296-5p.

miR-27a is significantly upregulated in HNSCC cell lines and its expression is correspondingly reduced by using knock dow $n$ anti sense approaches. ${ }^{22}$ It is considered a noncausal HNSCC miRNA as its manipulation in vitro does not have an effect on phenotype. ${ }^{17}$ miR-92a, previously named miR-92 in the miRNA database, has two precursor sequences: miR-92a- 1 and miR-92a- $2 .{ }^{23}$ miR-92a- 1 is located on the oncogenic miR-17-92 polycistron of chromosome 13q31.3, a locus that harbors quite a few upregulated miR N A s. ${ }^{22}$ mir-92a-2, located on chromosome $X q 26.2$, belongs to the miR-106a-92 cluster and is also overexpressed in HNSCC. ${ }^{22}$ miR-16 is commonly downregulated due to deletion of the $13 q 14$ region in chronic lymphocytic leukemia; ${ }^{24,25}$ however, it is significantly upregulated in HNSCC. ${ }^{22}$ The let-7 family of miRNA is known as a tumor suppressor miR NA that functions through inhibiting oncogenic mRNAs, such as RASA1, MYC, HMGA2. ${ }^{21,26,27}$ The let-7 family members are commonly downregulated in HNSCC except for let-7i which is upregulated. ${ }^{10,22,28}$ L ow levels of both let- $7 \mathrm{~d}$ and miR-205 have been reported to be significantly related to poor survival in primary HNSCC. ${ }^{11}$

miR-31, dysregulated in many cancers, ${ }^{29-32}$ appears to favor upregulation in $\mathrm{HNSCC}^{14}$ and oral squamous cell carcinoma $(\mathrm{OSCC})^{6,16}$ cell lines. Its function in tumorigenesis is unclear; however, Liu et al ${ }^{33}$ found miR-31 to be

Table 2: qPCR confirmation of qNPA assay

\begin{tabular}{llllll}
\hline & miR-31 & miR-193b & miR-663b & miR-923 & miR-1826 \\
\hline LT-ID6 & Up & Up & Up & Down & Down \\
LT-ID8 & Up & Up & Down & Down & Up \\
LT-ID9 & Up & Up & Up & Up & Up \\
LT-ID10 & Up & Up & Up & Down & Down \\
LT-ID11 & Up & Up & Up & Down & Down \\
LT-ID12 & Up & Up & Up & Down & Down \\
LT-ID13 & Up & Up & Up & Up & D \\
LT-ID14 & Up & Up & Up & Up & Down \\
LT-ID15 & Up & Up & Down & Down & $6 / 9$ down \\
\hline
\end{tabular}

\footnotetext{
${ }^{\Delta \Delta} C_{t}$ relative quantization (tumor ${ }^{\Delta} \mathrm{C}-$ normal ${ }^{\Delta} \mathrm{C}$ ) used to determine up or downregulation
} 
significantly elevated in the plasma of OSCC patients, which was remarkably reduced following surgery. This suggests that miR-31 may be an oncogenic miR NA. Its detection in plasma could be clinically useful as a noninvasive diagnostic approach. miR-296 is downregulated in OSCC. ${ }^{6}$

Several studies have reported significant differential expression of $\mathrm{miR}-100$, $\mathrm{miR}-125 \mathrm{~b}$ and $\mathrm{miR}-375$ in HNSCC, ${ }^{5,13,14,16,34}$ none of which reached significance in this study.

Of the 23 significantly differentially expressed miR NAs, 15 have not been previously reported in HNSCC and include: miR-663b, miR-663, miR-193b, miR-1291, miR720, miR-191, miR-1224-3p, miR-214, miR-1285, miR 1207-5p, miR -483-5p, miR-1225-3p, miR - 1228 , miR - 1280 and $\mathrm{miR}-638$.

Dysregulation of miR-193b is thought to influence melanoma development ${ }^{35}$ via its role of cell proliferation repression and regulation of CCND1 expression. ${ }^{35}$ In prostate cancer, miR -193b can present as an epigenetically silenced putative tumor suppressor. ${ }^{36}$ It has also been detected in cervical cancer cell lines. ${ }^{37}$ The role of miR $663 \mathrm{~b}$ reported in the development of human leukemias ${ }^{24}$ and of miR-663 in human colorectal cells ${ }^{38}$ is not known.

miR-191 is upregulated in hepatocellular carcinoma (HCC) and affects the TGF- $\beta$ and MAPK pathways, which play an important role in HCC tumorigenesis. ${ }^{39,40}$ Inhibition of miR-191 by 2-O-metoxyethyl (M OE) anti-miR was found to decrease cell proliferation and induce apoptosis in vitro with significant reduction of tumor mass in an invivo mouse model of HCC. Therefore, in HCC, miR-191 may signal a potential therapeutic target.

miR-1224-3p has been identified as a mammalian mirtron which is a short hairpin intron that is a precursor for miR NA biogenesis. ${ }^{41} \mathrm{M}$ irtrons were originally found in invertebrates but are now known to exist in mammals also. miR-214 has been detected in melanomas, cervical and ovarian cancers. ${ }^{42-44}$ In melanomas, miR-214 suppresses TFAP2C leading to tumor progression. ${ }^{42} \mathrm{miR}-1285$ has been shown to inhibit expression of TP53. ${ }^{45}$ miR-1207-5p is expressed abundantly in colon cancer cell lines along with miR-1207-3p, and Northern blot data suggested a common promoter and transcriptional regulatory unit making these likely complimentary overlapping miR NAs. ${ }^{46}$ miR-483-5p is significantly upregulated in malignant adrenocortical carcinoma (ACC) as compared with benign tumors. ${ }^{47}$ Its expression can accurately distinguish tumors as benign or malignant.

miR - 638 has been detected in human colorectal cells ${ }^{38}$ and in gastric cancer, ${ }^{48}$ where it is significantly downregulated. It is present in a stable form in human plasma. Tanaka et al. ${ }^{49}$ found that the ratio of miR-92a/
miR-638 in plasma is useful for distinguishing leukemia patients from healthy individuals.

\section{CONCLUSION}

The human miRNA database continues to get fine tuned with respect to new additions as well as exclusions. This pilot study identified previously unreported miRNAs in LSCC that upon further investigation in larger studies may have clinical utility in LSCC. Our 23 aberrantly expressed miR N A s in LSCC, including the 15 unreported in HNSCC, require further examination with subsequent validation in larger cohorts for clinical relevance as LSCC-specific markers.

\section{ACKNOWLEDGMENT}

Drs Chen and W orsham had full access to all the data in the study and take responsibility for the integrity of the data and the accuracy of the data analysis. This study was supported by R01 NIH DE 15990 (Dr W orsham).

\section{REFERENCES}

1. B artel DP. M icroR NA s: Genomics, biogenesis, mechanism and function. Cell 2004;116:281-97.

2. Bartel DP. M icroR NAs: Target recognition and regulatory functions. Cell 2009;136:215-33.

3. Lee Y S, Dutta A. M icroR N A s in cancer. A nnu R ev Pathol 2009; 4:199-227.

4. Christensen BC, M oyer BJ , A vissar M, et al. A let-7 microR NA binding site polymorphism in the KRAS 3' UTR is associated with reduced survival in oral cancers. Carcinogenesis 2009; 30:1003-07.

5. Henson BJ, Bhattacharjee S, O'D ee DM, Feingold E, Gollin SM. Decreased expression of miR-125b and miR-100 in oral cancer cells contributes to malignancy. Genes Chromosomes Cancer 2009;48:569-82.

6. Kozaki K, Imoto I, M ogi S, Omura K, Inazawa J. Exploration of tumor-suppressive microRNAs silenced by DNA hypermethylation in oral cancer. Cancer R es 2008;68:2094-105.

7. Calin GA. M icroRNAs and cancer: What we know and what we still have to learn. Genome M ed 2009;1:78.

8. Garzon R, Calin GA, Croce CM . M icroR N A s in Cancer. A nnu Rev Med 2009;60:167-79.

9. Li J , H uang $H$, Sun L, et al. M iR-21 indicates poor prognosis in tongue squamous cell carcinomas as an apoptosis inhibitor. Clin Cancer Res 2009;15:3998-4008.

10. Chang SS, Jiang WW, Smith I, et al. MicroR NA alterations in head and neck squamous cell carcinoma. Int J Cancer 2008; 123:2791-97.

11. Childs G, Fazzari M, Kung G, et al. Low-level expression of microR N A s let-7d and miR-205 are prognostic markers of head and neck squamous cell carcinoma. Am J Pathol 2009;174: 736-45.

12. Cervigne NK, Reis PP, M achado J, et al. Identification of a microRN A signature associated with progression of leukoplakia to oral carcinoma. Hum M ol Genet 2009;18:4818-29. 
13. A vissar M, Christensen BC, K elsey KT, M arsit CJ. M icroR NA expression ratio is predictive of head and neck squamous cell carcinoma. Clin Cancer Res 2009;15:2850-55.

14. Tran $N, M C L$ ean $T$, Zhang $X$, et al. MicroRNA expression profiles in head and neck cancer cell lines. Biochem Biophys Res Commun 2007;358:12-17.

15. Park NJ, Zhou H, Elashoff D, et al. Salivary microR NA: Discovery, characterization and clinical utility for oral cancer detection. Clin Cancer Res 2009;15:5473-77.

16. Wong TS, Liu XB, W ong BY, Ng RW, Y uen AP, Wei WI. $M$ ature miR-184 as potential oncogenic microR $A$ of squamous cell carcinoma of tongue. Clin Cancer Res 2008; 14:2588-92.

17. Babu JM, Prathibha R, Jijith VS, Hariharan R, Pillai M R. A miR-centric view of head and neck cancers. Biochim Biophys Acta 2011;1816:67-72.

18. M artel RR, Botros IW, Rounseville MP, et al. Multiplexed screening assay for mRN A combining nuclease protection with luminescent array detection. Assay Drug Dev Technol 2002; 1:61-71.

19. Johnson G. Cancer's Secrets Come Into Sharper Focus New Y ork Times, 2011.

20. Cimmino A, Calin GA, Fabbri $M$, et al. miR-15 and miR-16 induce apoptosis by targeting BCL 2. Proc N atl A cad Sci USA 2005;102:13944-49.

21. LeeY S, Dutta A. Thetumor suppressor microR NA let-7 represses the HM GA 2 oncogene. Genes Dev 2007; 21:1025-30.

22. Hui $A B$, Lenarduzzi $M, K$ rushel $T$, et al. Comprehensive MicroRNA profiling for head and neck squamous cell carcinomas. Clin Cancer Res 2010; 16:1129-39.

23. Mourelatos Z, Dostie J, Paushkin S, et al. miRNPs: A novel class of ribonucleoproteins containing numerous microRNAs. Genes Dev 2002;16:720-28.

24. Takada S, Y amashita Y, Berezikov E, et al. M icroR NA expression profiles of human leukemias. Leukemia 2008; 22:1274-78.

25. Calin GA, Dumitru CD, Shimizu M, et al. Frequent deletions and down-regulation of microR N A genes miR 15 and miR 16 at $13 q 14$ in chronic lymphocytic leukemia. Proc Natl A cad Sci USA 2002;99:15524-29.

26. J ohnson SM, Grosshans H, Shingara J, et al. RA S is regulated by the let-7 microRNA family. Cell 2005;120:635-47.

27. Sampson VB, Rong NH, Han J, et al. M icroRNA let-7a downregulates $M Y C$ and reverts $M Y C$-induced growth in Burkitt lymphoma cells. Cancer Res 2007;67:9762-70.

28. Ramdas L, Giri U, A shorn CL, et al. miR N A expression profiles in head and neck squamous cell carcinoma and adjacent normal tissue. Head Neck 2009;31:642-54.

29. Wong $Q W$, Lung RW, Law PT, et al. M icroRNA-223 is commonly repressed in hepatocellular carcinoma and potentiates expression of Stathmin. Gastroenterology 2008;135:257-69.

30. Slaby 0 , Svoboda M, Fabian $P$, et al. Altered expression of miR-21, miR-31, miR-143 and miR-145 is related to clinicopathologic features of colorectal cancer. Oncology 2007; 72:397-402.

31. Zhang $Y, G$ uo J, Li D, et al. Down-regulation of miR-31 expression in gastric cancer tissues and its clinical significance. Med Oncol 2010;27:685-89.

32. Schaefer $A$, Jung $M, M$ ollenkopf $H J$, et al. Diagnostic and prognostic implications of microRNA profiling in prostate carcinoma. Int J Cancer 2010;126:1166-76.
33. Liu CJ, K ao SY, Tu HF, T sai M M, Chang K W, L in SC. Increase of microR NA miR-31 level in plasma could be a potential marker of oral cancer. Oral Dis 2010;16:360-64.

34. $Y u T, W$ ang $X Y, G$ ong $R G$, et al. The expression profile of microRNAs in a model of 7,12-dimethyl-benz[a]anthranceinduced oral carcinogenesis in Syrian hamster. J Exp Clin Cancer Res 2009;28:64.

35. Chen J, Feilotter HE, Pare GC, et al. M icroR NA -193b represses cell proliferation and regulates cyclin D1 in melanoma. A m J Pathol 2010;176:2520-29.

36. Rauhala HE, Jalava SE, Isotalo J, et al. miR-193b is an epigenetically regulated putative tumor suppressor in prostate cancer. Int J Cancer 2010;127:1363-72.

37. L ui W O, Pourmand N, Patterson BK, Fire A. Patterns of known and novel small RNAs in human cervical cancer. Cancer Res 2007;67:6031-43.

38. Cummins JM, He Y, Leary RJ, et al. The colorectal microRN A ome. Proc N atl A cad Sci USA 2006; 103:3687-92.

39. Elyakim E, Sitbon E, Faerman A, et al. hsa-miR-191 is a candidate oncogene target for hepatocellular carcinoma therapy. Cancer Res 2010;70:8077-87.

40. Calvisi DF, Pascale RM , Feo F. Dissection of signal transduction pathways as a tool for the development of targeted therapies of hepatocellular carcinoma. Rev Recent Clin Trials 2007;2: 217-36.

41. Berezikov E, Chung WJ, Willis J, Cuppen E, Lai EC. Mammalian mirtron genes. M ol Cell 2007;28:328-36.

42. Penna E, Orso F, Cimino D, et al. M icroR NA-214 contributes to melanoma tumour progression through suppression of TFA P2C. EM BO J 2011;30:1990-2007.

43. Qiang $R, W$ ang $F$, Shi $L Y$, et al. Plexin-B 1 is a target of mi $R$ 214 in cervical cancer and promotes the growth and invasion of HeL a cells. Int J Biochem Cell Biol 2011;43:632-41.

44. $Y$ in $G$, Chen $R, A$ Ivero $A B$, et al. TWISTing stemness, inflammation and proliferation of epithelial ovarian cancer cells through M IR 199A 2/214. Oncogene 2010;29:3545-53.

45. Tian S, Huang S, W u S, Guo W, Li J, He X. M icroR NA - 1285 inhibits the expression of p53 by directly targeting its $3^{\prime}$ untranslated region. Biochem Biophys Res Commun 2010; 396:435-39.

46. Huppi K, Volfovsky N, Runfola T, et al. The identification of microRNAs in a genomically unstable region of human chromosome 8q24. M ol Cancer Res 2008;6:212-21.

47. Patterson EE, Holloway AK, Weng J, Fojo $T$, K ebebew E. M icroR NA profiling of adrenocortical tumors reveals miR-483 as a marker of malignancy. Cancer 2011;117:1630-39.

48. $Y$ ao $Y$, Suo $A L$, Li ZF, et al. M icroRNA profiling of human gastric cancer. Mol M ed Report 2009;2:963-70.

49. T anaka M, Oikawa K, Takanashi $M$, et al. D own-regulation of miR-92 in human plasma is a novel marker for acute leukemia patients. PL OS One 2009; 4:e5532.

\section{ABOUT THE AUTHORS}

\section{Kang Mei Chen}

Department of $\mathrm{O}$ tolaryngology/H ead and $\mathrm{N}$ eck R esearch, $\mathrm{H}$ enry Ford Hospital, MI, USA 


\section{Josena K Stephen}

Research Scientist, Department of Otolaryngology/Head and Neck Research, Henry Ford Hospital, MI, USA

\section{Shaleta Havard}

Senior Research A ssistant, D epartment of Otolaryngology/Head and Neck R esearch, Henry Ford Hospital, MI, USA

\section{Veena Shah}

Pathologist, Department of Pathology, Henry Ford Hospital, M I USA

\section{Glendon Gardner}

Otolaryngologist, Department of Otolaryngology/Head and Neck Surgery, Henry Ford Hospital, M I, USA

\section{Vanessa G Schweitzer}

Otolaryngologist, Department of Otolaryngology/Head and Neck Surgery, Henry Ford Hospital, M I, USA

\section{Maria J Worsham (Corresponding Author)}

FACM G, Director of R esearch, Department of Otolaryngology/H ead and Neck Research, Henry Ford Hospital, I Ford Place, ID, Detroit MI 48202, USA, Phone: 313-874-3350, Fax: 313-874-1079 e-mail: mworsha1@hfhs.org 